\title{
A low-cost multi-channel aerosol fluorescence sensor for networked deployment
}

\author{
Paul H Kaye ${ }^{\mathrm{a}}$, Edwin Hirst ${ }^{\mathrm{a}}$, Virginia Foot ${ }^{\mathrm{b}}$, James M Clark ${ }^{\mathrm{b}}$, and Karen Baxter ${ }^{\mathrm{b}}$. \\ ${ }^{\mathrm{a}}$ Science \& Technology Research Institute, University of Hertfordshire, Hatfield AL10 9AB, UK. \\ ${ }^{\mathrm{b}}$ Defence Science \& Technology Laboratory, Porton Down, Salisbury, Wilts. SP4 0JQ, UK.
}

\begin{abstract}
We describe a low-cost prototype bio-aerosol fluorescence sensor designed for unattended deployment in medium to large area networks. The sensor uses two compact xenon flash units to excite fluorescence in an aerosol sample volume drawn continuously from the ambient environment. In operation, the xenons are pulsed alternately at 300ms intervals whilst absorption filters restrict their radiation output to UV bands $\sim 260-290 \mathrm{~nm}$ and $\sim 340-380 \mathrm{~nm}$ respectively, optimal for exciting the biological fluorophores tryptophan and NADH. Fluorescence from all particles instantaneously present within a sensing volume is measured using two miniature photomultiplier detectors optically filtered to detect radiation in the bands $\sim 320-600 \mathrm{~nm}$ and $\sim 410-600 \mathrm{~nm}$. The second of these bands covers the principal emission from NADH, whilst the difference between the first and second detector channels yields fluorescence in the 320-410nm band, covering much of the tryptophan emission. Whilst each sensor is clearly limited in specificity, the low sensor cost $(<\$ 5 \mathrm{k})$ offers potential for the deployment in large networks that would be prohibitively expensive using particle fluorescence sensors based on currently available UV lasers. Preliminary details are also given of a variant of the sensor, currently under development, in which xenon illumination is used to acquire single particle fluorescence data at rates of up to 200 particles per second.
\end{abstract}

Keywords: Bio-aerosol, fluorescence, xenon, sensor, network.

\section{INTRODUCTION}

In the search for methods by which an ambient environment can be continuously monitored for potentially harmful biological aerosols, particle fluorescence methods have received considerable attention over the past decade. When excited by radiation tuned to the principal biological fluorophores contained within biological organisms, intrinsic particle fluorescence can be used to help differentiate biological from non-biological particles, and may even provide some discrimination between biological particles which are normal constituents of an ambient environment and those which may be considered a threat.

Because intrinsic fluorescence from biological fluorophores is generally weak, and because the fluorophores are normally present in extremely small quantities in airborne biological particles, the exciting radiation must be intense, and lasers have commonly been employed for this purpose. In the earliest systems, continuous wave lasers were employed (for example ${ }^{1,2}$ ) though these were usually large and operated at wavelengths which were too long for efficient excitation of some of the important bio-fluorophores such as tryptophan for which optimal excitation occurred at wavelengths of $\sim 260-280 \mathrm{~nm}$. Hence the use of solid-state lasers employing harmonic generation, such as frequency quadrupled Nd-YAG lasers, has gained acceptance (for example ${ }^{3,4,5}$ ), both for the output wavelength of $266 \mathrm{~nm}$ and because they offered a smaller form-factor than continuous-wave gas lasers.

These developments helped establish the potential of laser-induced fluorescence (LIF) for the characterization of single airborne particles and the detection of potential pathogens. However, solid-state harmonic lasers are expensive and comparatively fragile components to be incorporated in systems where multiple-point detection or widespread field monitoring may be required. Because of this, considerable effort is presently going into the development of compact and robust semiconductor sources capable of delivering continuous wave sub-300nm radiation. In particular, the US SUVOS (Semiconductor Ultraviolet Optical Sources) ${ }^{6}$ programme, which commenced in 2002, is making significant 
gains towards this objective and has already demonstrated prototype LED (light emitting diode) devices capable of room temperature continuous 280nm emission at milliwatt power levels. These devices have already been incorporated in a test-bed sensor for bioaerosols ${ }^{7}$.

UV LEDs and ultimately UV diode lasers (which present greater technical challenges but are capable of higher irradiances) undoubtedly offer the best prospects for implementing LIF in low-cost bio-aerosol sensors. However, before their routine incorporation in such devices, issues such as limited device lifetimes, currently $<1,000$ hours for LEDs, must be overcome. In the meantime, alternative low-cost sources of UV still have value, and this paper describes a prototype bio-aerosol fluorescence sensor based on compact xenon sources.

\section{DUAL XENON-BASED SENSOR}

The prototype described here, referred to as WIBS (Wide Issue Bio-aerosol Sensor), was developed with the selfimposed constraint of using only low-cost COTS (commercial off-the-shelf) components. In addition, we required a sensor which would provide at least two fluorescence excitation wavebands and two fluorescence emission detection wavebands tuned to the tryptophan and NADH emission spectra. The fluorescence excitation-emission matrix would then be the basis for aerosol characterization.

Miniature xenon discharges tubes, similar to those found in the ubiquitous disposable camera, are readily commercially available. As optical sources, they suffer the limitations of pulsed output, low pulse repetition rate (relative to pulsed lasers), and essentially isotropic emission. Nevertheless, compared to solid-state harmonic lasers they are very cheap and relatively efficient producers of UV radiation in the required $260-290 \mathrm{~nm}$ and 340-380 nm wavebands.

The xenon flash-lamp sources selected for use in the prototype were Perkin Elmer RSL3100 series ${ }^{9}$ because of their low cost, low power requirement (2W), small size, (8 x 4 x $2.5 \mathrm{~cm}$ approx.), and built-in quasi-collimation optics. They operated with a maximum flash energy (electrical) of $40 \mathrm{~mJ}$ and a flash duration of $1 \mu \mathrm{s}$. Although their maximum repetition rate was $50 \mathrm{~Hz}$, we envisaged operation at typically 2 - $3 \mathrm{~Hz}$ to achieve a useable xenon lifetime (quoted at $10^{8}$ flashes) of at least 12 months continuous operation. The quoted broad spectrum $(200-1100 \mathrm{~nm})$ fluence at $2.5 \mathrm{~cm}$ from the output window was $115 \mu \mathrm{J} / \mathrm{cm}^{2}$, of which $\sim 10 \%$ lay within the required $260-290 \mathrm{~nm}$ waveband and $~ 5 \%$ in the 340-380 nm waveband. When further focused to a 10mm diameter particle illumination beam (see section 2.1), the xenons were capable of delivering fluences of typically $\sim 15-20 \mu \mathrm{J} / \mathrm{cm}^{2}$ of useable UV to the particle sample.

Our own experience of particle fluorescence characterisation ${ }^{8}$, together with an analysis of some other existing fluorescence measurement systems ${ }^{1-5}$, allowed an indicative assessment of the fluence of excitation radiation that must be delivered to a typical biological particle of interest in order to produce a measurable fluorescence signal for a given solid angle of fluorescence collection (assuming isotropic emission) and a given breadth of spectral band. This established that a fluence of typically $\sim 300 \mu \mathrm{J} / \mathrm{cm}^{2}$ would be required if WIBS was to achieve single particle fluorescence sensitivity. Given that the available UV fluence was less than a tenth of this, it followed that necessarily WIBS would typically require a minimum of $\sim 15$ to 20 particles in the sensing volume at any one time. (Although integration of the fluorescence from a smaller number of particles illuminated by several flashes in rapid succession was possible, this would reduce the effective lifetime of the xenon).

Collecting fluorescence emission from several particles simultaneously would clearly compromise the specificity of the sensor. However, the philosophy behind WIBS was based on the concept of large number of networked sensors deployed throughout or surrounding a sensitive area, such that, whilst the information from individual sensors was limited, the collated information from all sensors could provide meaningful information on the presence and spatial behaviour of potentially harmful aerosols. (An alternative approach, based on xenon-excited fluorescence measurement from individual aerosol particles, is under development and is described later in this paper).

\subsection{Sensor Configuration}

Figure 1 shows schematic plan view of the WIBS dual-xenon sensor configuration. The sensor is arranged with the two alternately-pulsed xenon sources, Xenon 1 and Xenon 2, either side of a central scattering chamber. Orthogonal to the axis of the xenons are two fluorescence detector channels. These incorporate two apertured spherical glass mirrors 
(Edmund Optics T43-470) separated by their focal length (50mm), such that light emanating from particles near the centre of the chamber is reflected by one mirror through the aperture in the opposing mirror, as indicated in Fig.1. A suitable collection lens then concentrates this light through an optical filter (see below) and onto the photocathode of a miniature photomultiplier (PMT) detector module (Hamamatsu H6779).

The aerosol flow of approximately $10 \mathrm{l} / \mathrm{min}$ is drawn from the ambient environment through the central chamber of the sensor by a small electrical fan (not shown). No filtering or ensheathing of the aerosol flow takes place in order to minimise power requirements. The light pulse from each xenon source is optically filtered to the required spectral band (see below) and configured to provide a near-collimated beam of circular cross-section $\sim 10 \mathrm{~mm}$ diameter at the chamber mid-point. The optical filters employ reflective films and, to minimise unwanted UV light scatter in the chamber, are angled at $15^{\circ}$ to prevent back reflection from the opposing xenon source.

Although the aerosol enters the sensor body through a cylindrical tube of approximately $14 \mathrm{~mm}$ diameter, the central part of the chamber is configured in such a way as to convert this cylindrical flow to a

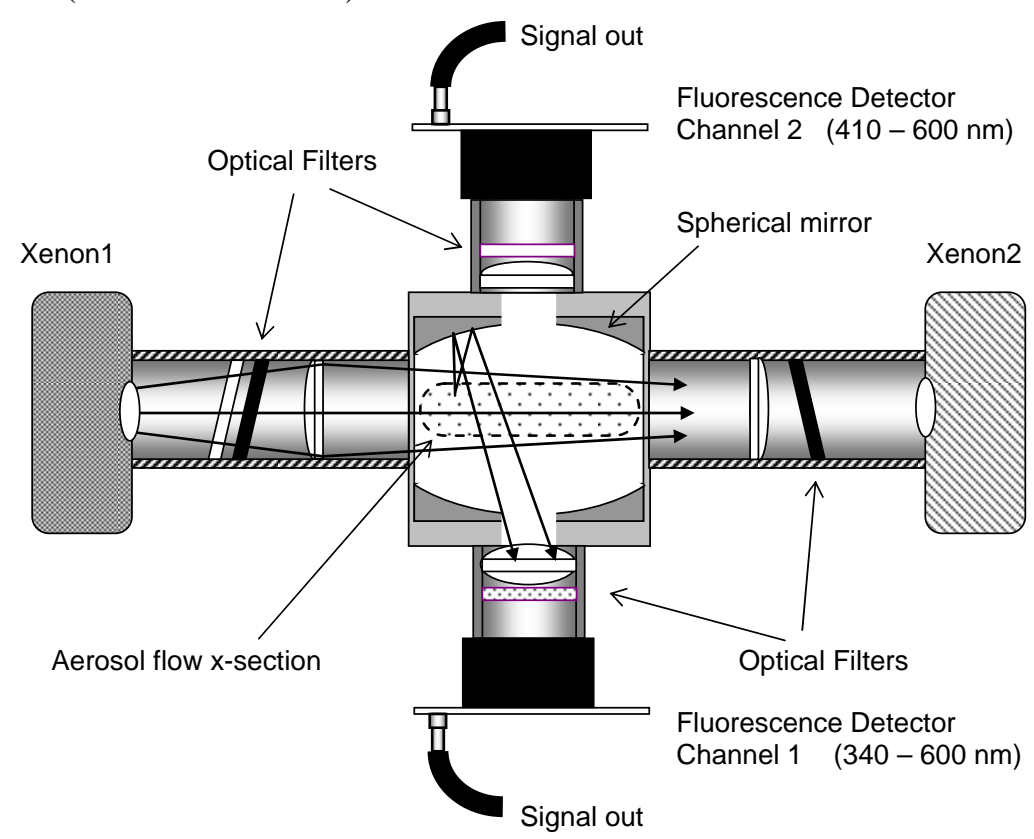

Fig. 1. Plan view of the layout of the WIBS sensor rectangular cross-section, approximately $8 \mathrm{~mm}$ by $50 \mathrm{~mm}$, as it traverses the illumination path from the xenons. This maximises the sensing volume, defined by the intersection of the aerosol flow and the illuminating UV beam, in an attempt to improve sensitivity for low concentration aerosols. The effective sensing volume is currently $\sim 4 \mathrm{ml}$. Fig.2 shows the assembled prototype sensor with its associated data acquisition electronics and power management.

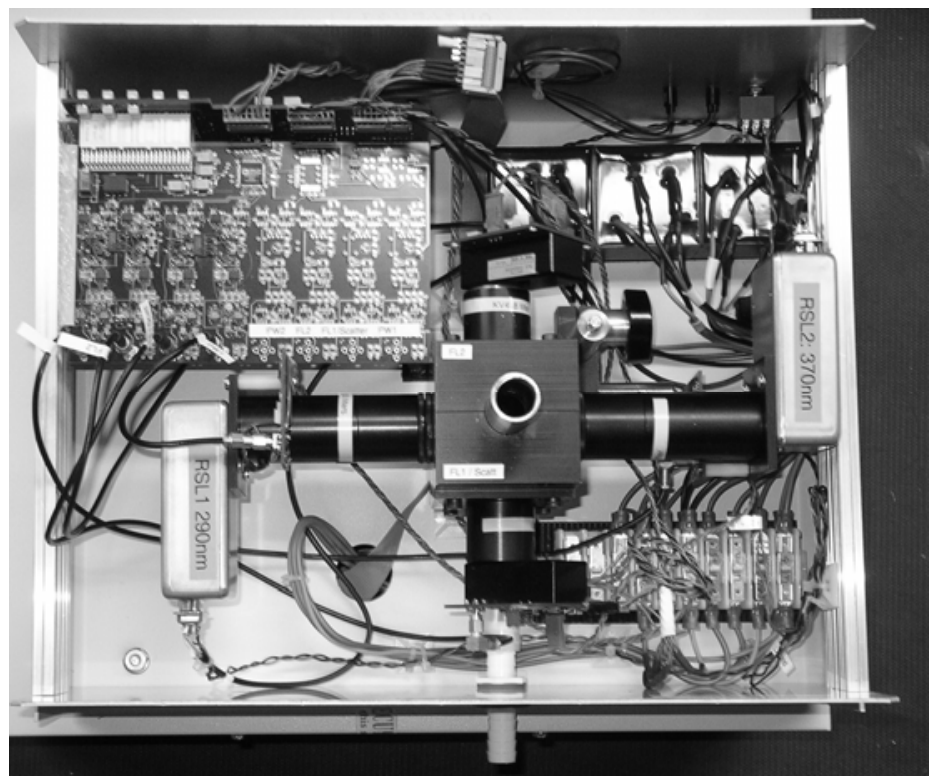

Fig. 2. WIBS sensor layout from above. 


\subsection{Excitation and Emission Filters}

Initially, standard COTS 25mm diameter optical filters were sought to define both the excitation and fluorescence detection bands of WIBS. Interference filters were avoided for reasons of relatively high cost, low transmittance, and requirement for normal incidence of the radiation. However, in the event, two custom filters became available whose performance surpassed that of their COTS equivalents, and these were employed (see below). Selection of filters had to meet other criteria in terms of high transmittance, minimum intrinsic filter fluorescence, and resistance to solarisation under prolonged UV illumination.

\subsubsection{Xenon 1 Excitation}

Excitation from the Xenon 1 is designed to coincide with the absorption peak of tryptophan at approximately 280nm. Originally, this excitation band was provided by a pair of filters in series. The first, a UVB290 filter from Korean manufacturer Daejin Special Optics Co. ${ }^{10}$, provided good transmittance in the 250-300nm band but also some unwanted transmittance in the $450-500 \mathrm{~nm}$ band. To remove the latter contribution in the excitation beam, a U-330 filter (Hoya Corporation) was used in series. The combined transmittance is illustrated in Fig. 3a opposite. Subsequently, this pair of filters was replaced with a single custom filter $^{11}$ which exhibited a similar transmittance curve but without any significant leakage in the visible spectrum.

Fig. 3a. Transmittance characteristic for Xenon 1 excitation.

\subsubsection{Xenon 2 Excitation}

Excitation from the Xenon 2 source is designed to exploit the absorption peak of NADH at around 340nm. Although custom filters could be produced to give a narrow transmittance peaking at this wavelength, the best COTS alternative was the DUG11 filter (Schott AG, Mainz, Germany). As shown opposite, this exhibits a wider transmittance band than would be ideal, but also has very good out-of-band blocking and has a high peak transmittance $(70 \%)$ at $335 \mathrm{~nm}$.

Fig. 3b. Transmittance characteristic for Xenon 2 excitation.

\subsubsection{Fluorescence 1 Detector Channel}

The Fluorescence 1 channel of WIBS is required to measure fluorescence across a band coinciding with the maximum of the emission spectrum of tryptophan, ie: approximately 300-420nm, peaking at $\sim 345 \mathrm{~nm}$. However, it was also imperative that the transmittance of the Fluorescence 1 channel did not overlap with that of the Xenon 1 excitation, shown in Fig.2a since this excitation extended, albeit very weakly, to $\sim 315 \mathrm{~nm}$. Originally, a UV-34 filter (Hoya Corporation) was employed, giving a high-pass beyond 360nm. However, this was relatively inefficient in the capture of potential tryptophan emission below 360nm, and a superior

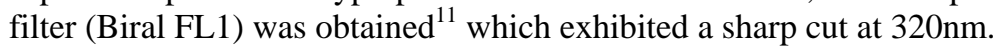

Fig. 3c. Transmittance characteristic for Fluorescence 1 Channel.
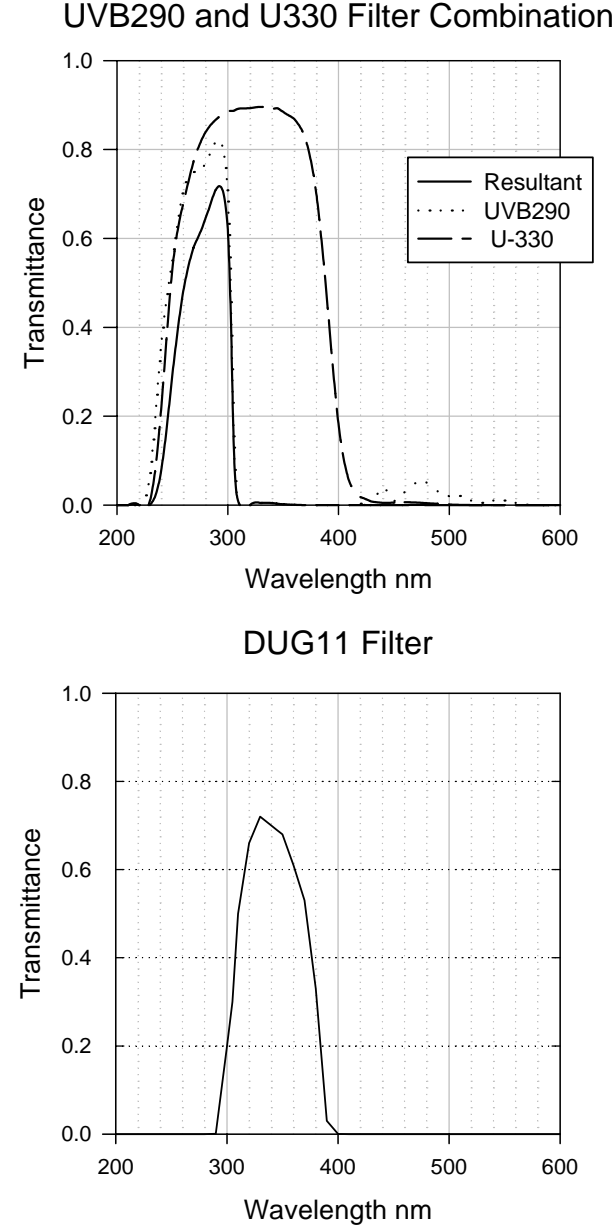

BIRAL FL1 and PMT Response

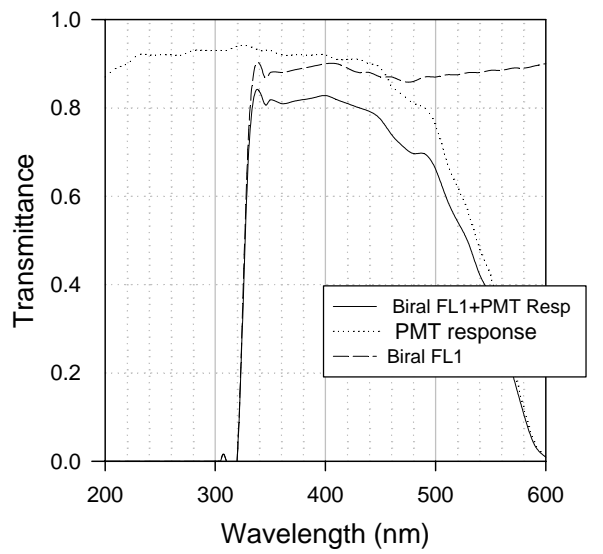


The combination of the transmittance of the Biral FL1 filter with the roll-off in responsivity of the photomultiplier photocathode produces a broad transmission band from 320nm to approximately 600nm, as in Fig.3c. Whilst this band extends far beyond the upper limit of the primary tryptophan emission peak at approximately 420nm, it is the difference between this transmission and that of the Fluorescence 2 Channel (see Fig. 4 below) that embraces the majority of the band associated with tryptophan emission.

\subsubsection{Fluorescence 2 Detector Channel}

The second WIBS fluorescence detection channel is targeted towards the NADH emission band, ie: 400-600nm, peaking at 450nm. This band definition was effectively achieved using a standard KV418 filter (Schott AG, Mainz, Germany), a high pass filter with sharp cut-off and very low intrinsic fluorescence. The combination of this filter's transmittance and the roll-off in responsivity of the photomultiplier photocathode effectively defined the required band, as in Fig.3d.

KV418 and PMT Responsivity

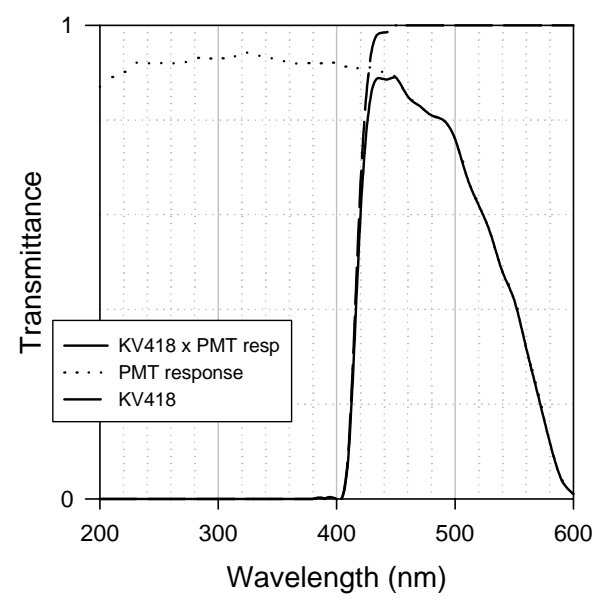

Fig. 3d. Transmittance characteristic for Fluorescence 1 Channel.

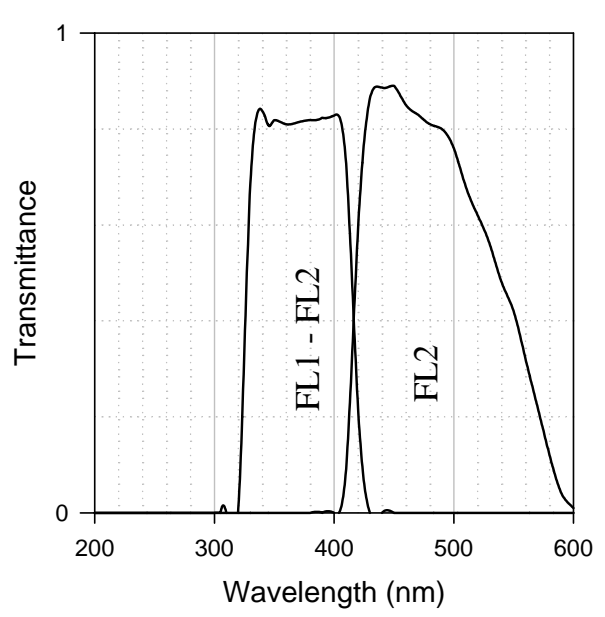

Fig.4. Transmission bands (FL1-FL2) and FL2 used in subsequent data analysis to assess potential tryptophan and NADH fluorescence respectively.

Finally, Fig.4 shows the two transmission bands (FL1-FL2) and FL2 which are used in subsequent data analysis to ascertain the level of particle fluorescence present in the wavebands associated with tryptophan and NADH respectively.

\subsection{Sensor Operation Sequence}

In operation, the two xenon sources, 1and 2, fire alternately at 300 ms intervals. For each xenon pulse, the fluorescence signals received by the FL1 and FL2 fluorescence channels are integrated electronically and digitized before being transmitted to a host computer for display and analysis (see below). In addition, the output pulse from each xenon source is measured using a UV-sensitive photodiode in order to allow correction for fluctuations in pulse-to-pulse energy (typically <3\%).

When the Xenon 2 source fires, the radiation emanating from its band-limiting filter (300-400nm) overlaps significantly with the detection band of the Fluorescence 1 channel (320nm-600nm). In effect, this means that the Fluorescence 1 channel will be responding to elastically scattered light from particles within the sensing volume rather than particle fluorescence (which would be orders of magnitude weaker). This elastic-scatter signal provides a crude estimate of the aerosol concentration within the sensing volume at the instant of the Xenon 2 flash. However, this must be treated with caution since the actual magnitude of the signal recorded will be a function of the particle size distribution as well as particle number - larger particles scattering proportionally more energy to low forward angles that would not be collected by the Fl2 channel. 
Since the elastically scattered signal recorded by the FL1 detector channel when Xenon 2 fires is normally significantly greater than the fluorescence signal recorded by that same channel when the Xenon 1 fires, it is necessary for the gain of the PMT detector to be changed according to which Xenon is firing. This is achieved by switching the level of the PMT gain automatically under computer control during each firing cycle of the two xenons.

\subsection{Data Display}

Whilst the intention is that in a multi-point deployment of WIBS sensors, raw data from each sensor would be sent by radio signal to a central processing unit, in the prototype testing, the host computer was used to record, analyse, and display results in real time. The user interface was developed using the VEE $^{\mathrm{TM}}$ virtual instrument environment (Hewlett-Packard Inc.). The display comprised two synchronous history plots, as in Fig.5., plus various other operational controls. The upper history plot displayed as a function of time the data being recorded by the FL1 and FL2 fluorescence channels when the Xenon 1 ('280nm excitation') fired, together with the Xenon 1 pulse energy. The lower plot showed similarly the data from the FL1 and FL2 channels when the Xenon 2 ('370nm excitation') fired, together with the Xenon 2 pulse energy.

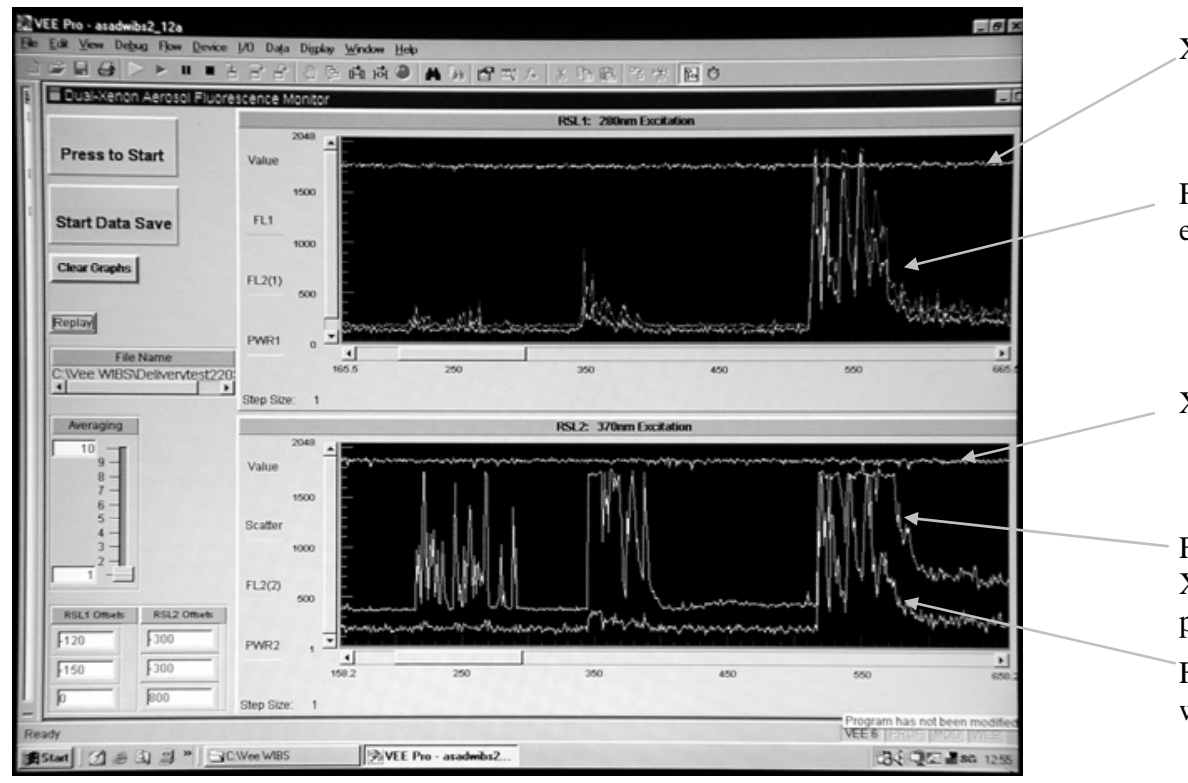

Xenon 1 pulse energy

FL1 and FL2 response to Xenon 1 excitation with aerosol present.

Xenon 2 pulse energy

FL1 (elastic scatter) response to Xenon 2 excitation with aerosol present.

FL2 response to Xenon 2 excitation with aerosol present.

Fig.5. User interface for WIBS prototype sensor.

\section{PRELIMINARY EXPERIMENTAL RESULTS}

Preliminary testing of the WIBS prototype took place at DSTL (Defence Science and Technology Laboratory), Porton Down, UK., in the spring of 2004. In these tests, various materials were and aerosolized from either dry powder or water suspension into a large ballast chamber (incorporating an air circulation fan to reduce sedimentation) using compressed-air powered nebulizers. The WIBS prototype was positioned directly below the ballast chamber and sampled the aerosol via a 20mm bore silicon rubber tube. An Aerodynamic Particle Sizer (Model 3320, TSI Inc., MN, USA) sampled simultaneously from the ballast chamber to allow data relating to particle size and concentration to recorded in parallel with the sensor fluorescence data.

An example of raw data recorded in this way over a period of approximately 30 minutes is shown in Fig.6. These raw data plots must be corrected for both level offsets (a result of dc electronic offsets produced in the integration circuitry) and intrinsic filter fluorescence arising from UV illumination of the filter materials. The former effect is readily measured and may be removed in data processing. The latter effect is manifest, for example, in the response of the 
WIBS sensor to $\mathrm{NaCl}$ aerosol. This material does not fluoresce and therefore no signal should be reported by the FL1 and FL2 detector channels. As can be seen from the $\mathrm{NaCl}$ data in Fig.6, traces (e) and (f) do report an unwanted response when Xenon 1 fires as a result of elastically scattered UV from the particles falling on the filters and producing intrinsic filter fluorescence. For each detector, this response is directly proportional to the level of elastic scatter, shown in trace (c), and may therefore be corrected for in the subsequent data processing.

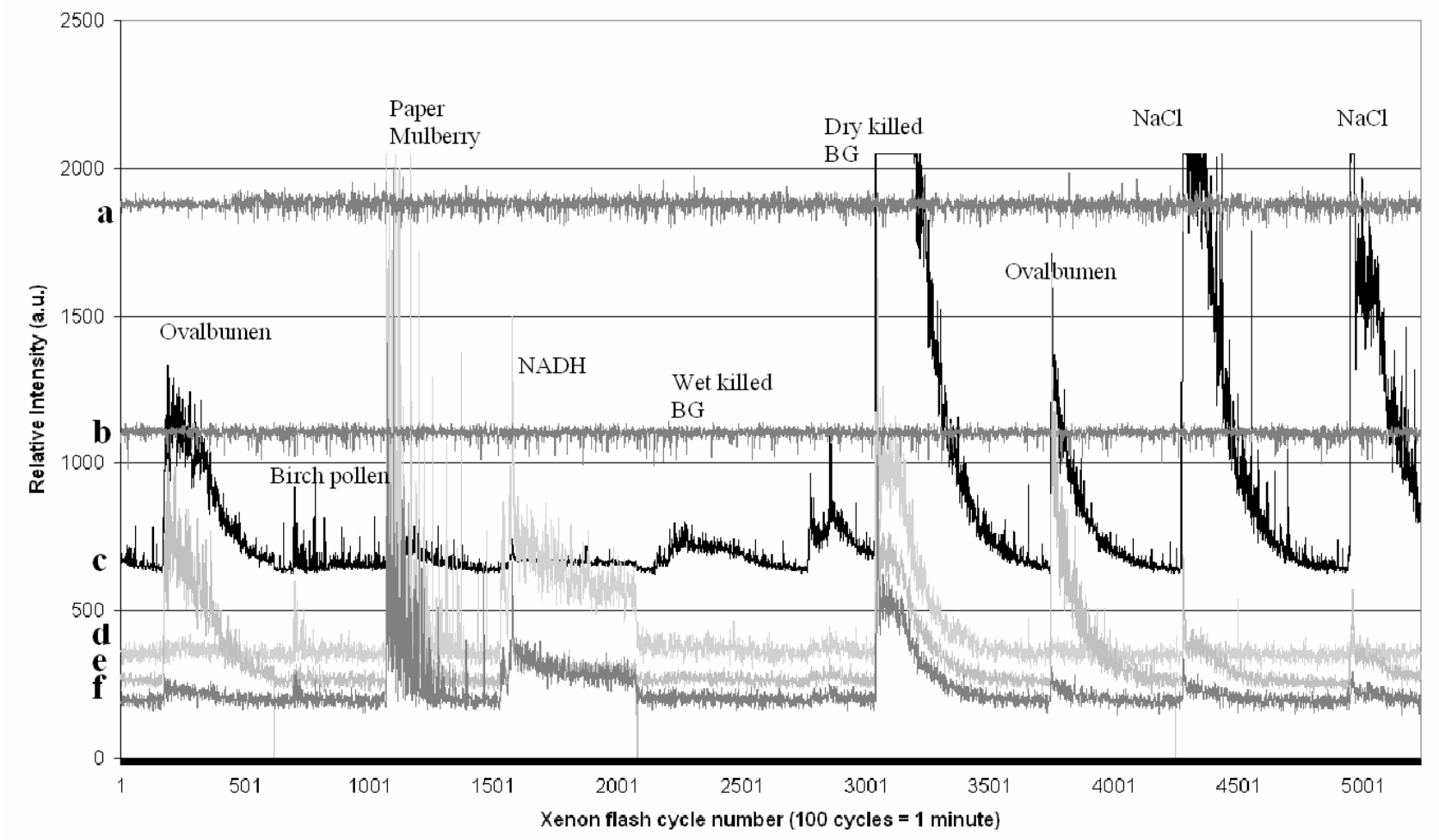

Fig.6. Raw data acquired from a variety of aerosols using the WIBS prototype sensor. Traces are: (a) Xenon 1 pulse energy; (b) Xenon 2 pulse energy; (c) FL1 response when Xenon 2 fires (ie: elastic scatter from particles); (d) FL2 response when Xenon 2 fires; (e) FL1 response when Xenon 1 fires; (f) FL2 response when Xenon 1 fires.

Fig.7 shows the same data as Fig.6 but corrected for offsets and intrinsic filter fluorescence. For clarity, the Xenon pulse energy traces (a) and (b), and the elastic scatter trace (c), are not shown. The first aerosol, ovalbumen, produces a significant fluorescence signal on the FL1 channel $(\sim 320-600 \mathrm{~nm})$ with Xenon 1 illumination $(\sim 260-290 \mathrm{~nm})$. This is to be expected as ovalbumen has similar fluorescence characteristics to those of tryptophan. For the same aerosol, the FL2 detector ( 410-600nm) shows much smaller response when the Xenon 1 fires, and virtually no signal at all when the Xenon 2 ( 300-400nm) fires, again as would be expected with this material.

In contrast, the NADH aerosol produces a maximum response in the FL2 channel ( 410-600nm) when the Xenon 2 fires, commensurate with the known fluorescence properties of this material which has an absorption peak at about $340 \mathrm{~nm}$ and a peak fluorescence at $\sim 450 \mathrm{~nm}$. Smaller but still measurable fluorescence is recorded by the FL2 and FL1 channels when the Xenon 1 fires, a result of the lower fluorescence efficiency at this shorter excitation wavelength. It is noticeable that the sensor responded strongly to the Dry Killed BG spores (Bacillus subtilis var niger) on both fluorescence channels and for both excitation wavebands. In contrast, the wet BG aerosol produced a far weaker response. This was due primarily to the disparity in concentrations produced in the dry and wet aerosols, confirmed by the Aerodynamic Particle Sizer. 


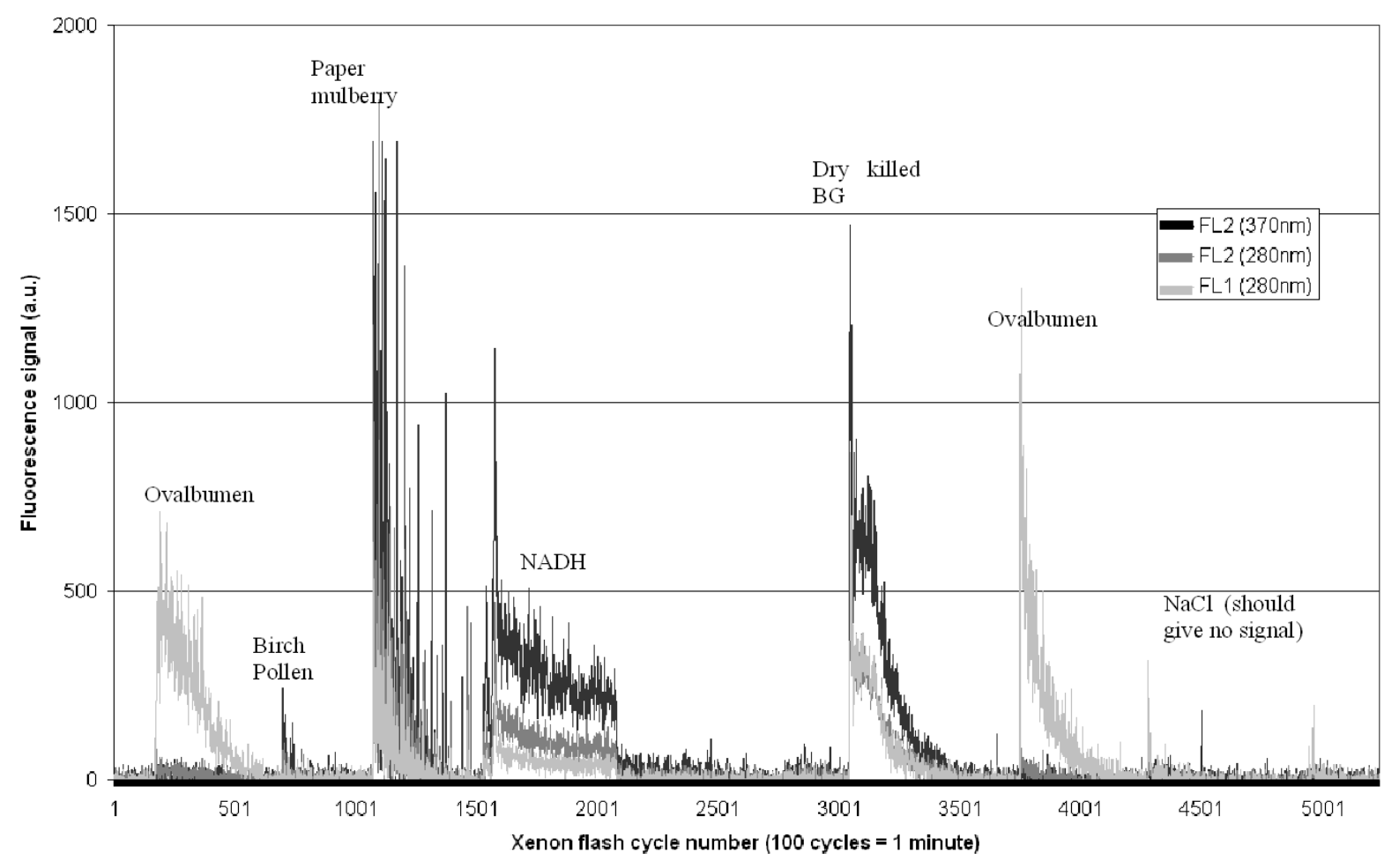

Fig.7. WIBS fluorescence data as shown in Fig.6 but after removal of offsets and correction for intrinsic filter fluorescence.

As stated, the proposed deployment of sensors such as WIBS would be in large networked arrays in which the collated aerosol fluorescence data would be processed by a central computer using, for example, artificial neural networks to analyse spatial aerosol properties and identify anomalous aerosol events. As a simplified example to indicate the degree to which the WIBS data may be used to help differentiate different aerosols, an analysis of the data in Fig.7 is shown in Fig.8. This shows, for four different aerosol types, the value of (FL1-FL2) for Xenon 1 ( 260-290nm) excitation as a function of the FL2 response under Xenon $2(\sim 300-400 \mathrm{~nm})$ excitation. The (FL1-FL2) data correspond to fluorescence in the band approximately 320-410nm.

\section{DISCUSSION}

Fig. 8 shows that, for these albeit pure aerosol types, reasonable differentiation is provided by this simple comparison of FL1 and FL2 responses. Clearly, environmental aerosols would normally contain a variety of constituent particle types, some in potentially very low concentrations, and interpreting the data from these aerosols would present a far greater challenge. Both laboratory and field testing of the WIBS prototype is currently underway with more complex aerosols, including common interferent aerosols such as diesel mist or aviation fuel mist. These tests will establish the performance parameters for a single sensor and, following the construction of additional sensor units, will underpin investigations into the monitoring and aerosol characterisation capabilities of networked sensors.

In the meantime, modifications to the current sensor design are underway to improve sensitivity to low aerosol concentrations. These involve changes in the twin mirror collection optics that will significantly increase the efficiency of capture of fluorescence emissions from particles, and modification to the central sensing chamber design to facilitate easier routine cleaning and replacement of xenon sources without need for re-alignment. 


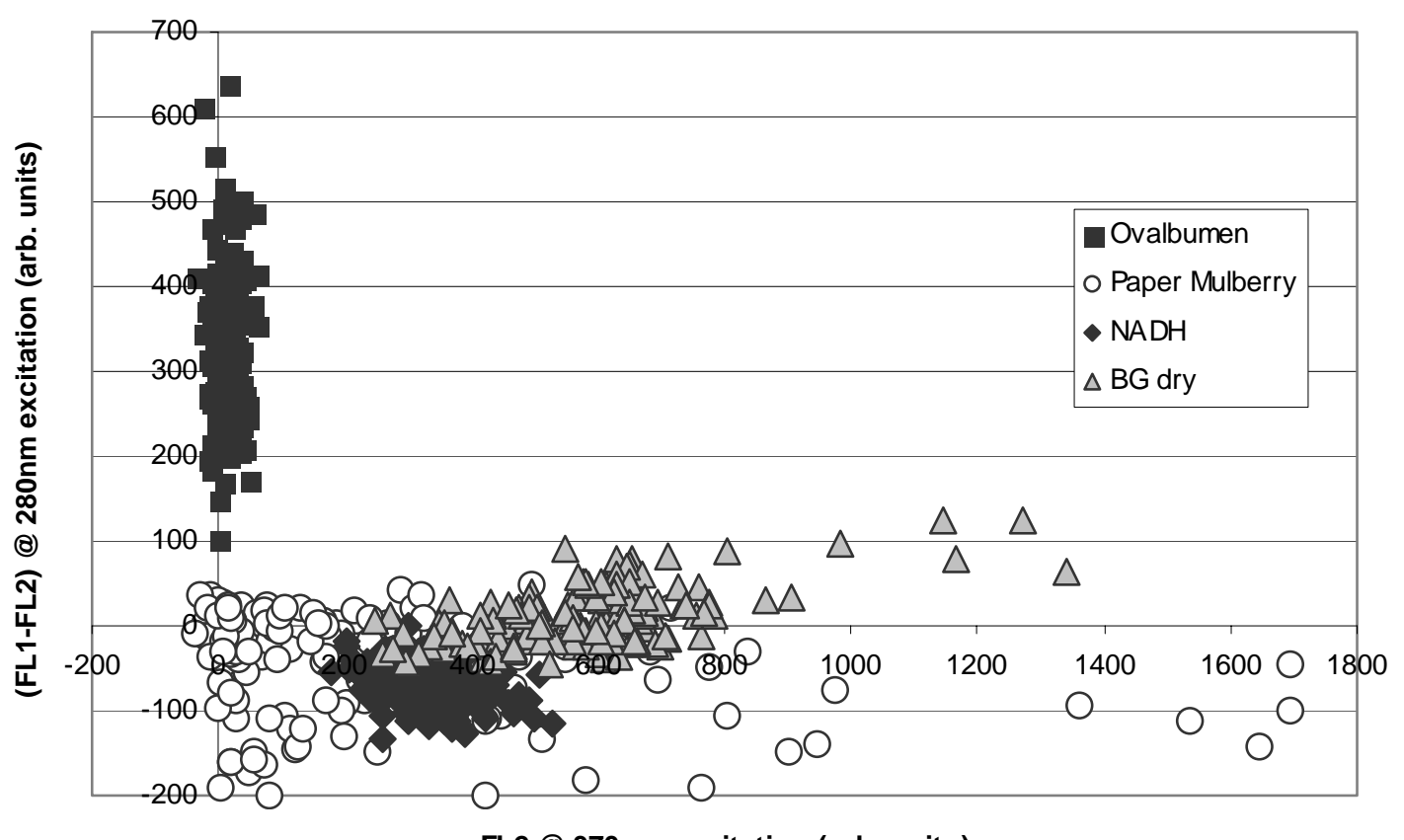

FL2 @ 370nm excitation (arb. units)

Fig.8. Analysis of four aerosol types based on the difference in response of the FL1 and FL2 detection channels under Xenon 1 excitation versus the response of the FL2 channel under the longer waveband Xenon 2 excitation.

\section{APPENDIX - SINGLE-PARTICLE WIBS VARIANT}

The WIBS prototype aerosol fluorescence sensor has been designed to record the fluorescence from a collection of particles within the instrument sensing volume using a comparatively low xenon pulse repetition rate of $\sim 1.6 \mathrm{~Hz}$. This approach maximized the xenon lifetime at the cost of reduced detection specificity.

An alternative approach, currently being developed, retains the use of xenon UV sources but is designed to examine single particle intrinsic fluorescence at peak rates of up to 200 particles/second peak and 'steady-state' rates of typically 50 particles/second. This development has been aided in part by recent advances in lifetimes of compact xenon lamps, which now exceed $10^{9}$ flashes. These xenons would provide at least 6 months ( 5,000 hours) of continuous 24/7 operation at the analysis rates mentioned above.

The single-particle fluorescence sensor is shown schematically in Fig. 9. In operation, particles are delivered in single file in an airflow column of approximately $0.5 \mathrm{~mm}$ diameter through the beam from a modulated (for reason, see below) continuous-wave diode laser $635 \mathrm{~nm}$ wavelength. The beam dimensions at the point of intersection with the sample airflow are approximately $2 \mathrm{~mm}$ width by $120 \mu \mathrm{m}$ depth. The time-of-flight of the particle through the beam is approximately $10 \mu \mathrm{s}$. Elastically scattered light from each particle is collected via an ellipsoidal reflector and directed through an iris onto a long wave pass dichroic beam-splitter whose cut wavelength is centred on $400 \mathrm{~nm}$. The transmitted scattered light subsequently light passes through a Biral FL1 filter ${ }^{11}$ and falls onto the photomultiplier detector of Fluorescence Detector Channel 2 (FL2). 


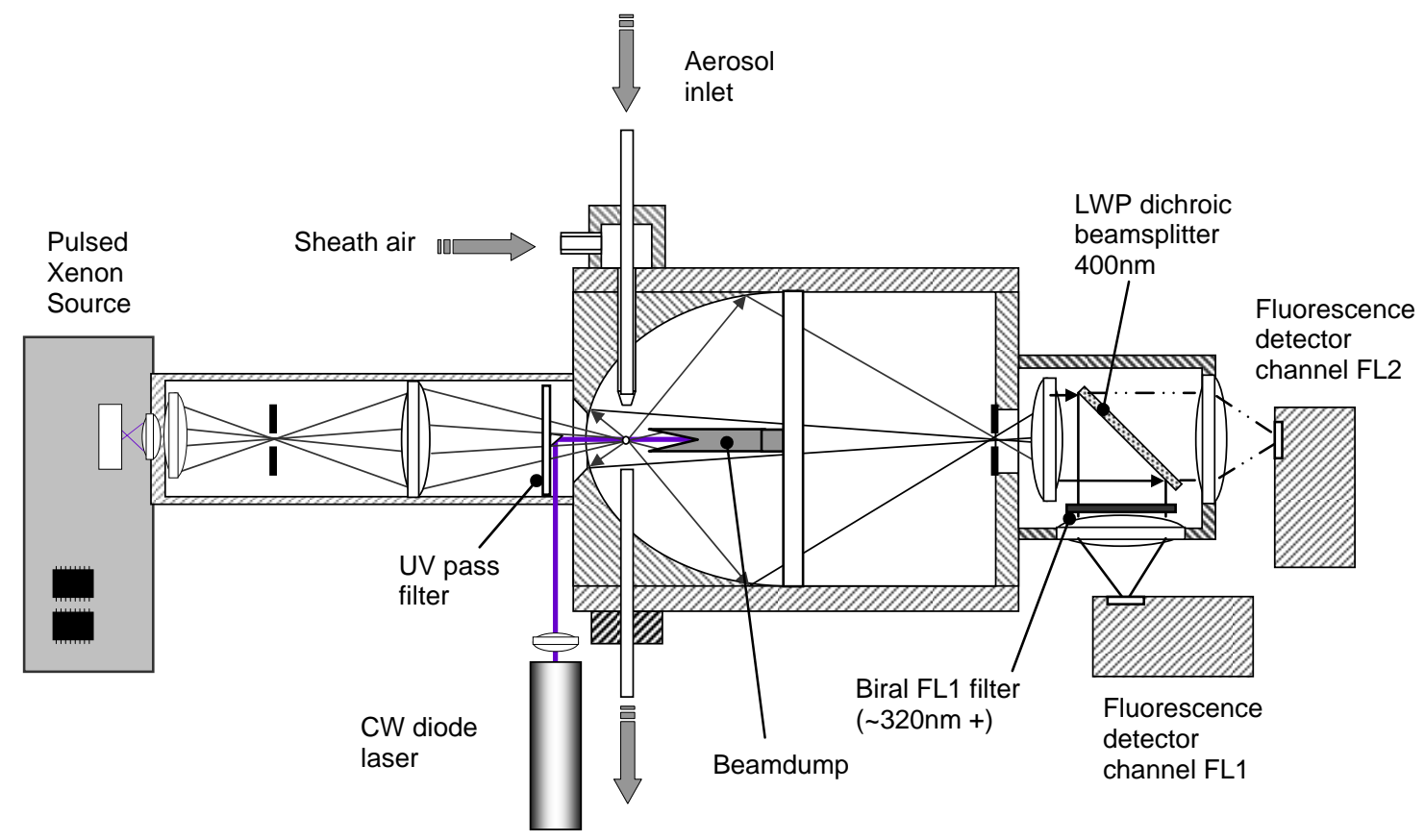

Fig.9. Schematic of single particle fluorescence prototype.

The detected elastic scatter signal from FL2 is of approximately Gaussian form as in Fig.10. As the falling edge of the signal passes through a pre-set threshold (which may be use to set an approximate minimum particle size to be excited with UV radiation), a trigger sequence is initiated which results in the firing of the xenon source approximately $5 \mu \mathrm{s}$ later, during which time the particle will have moved approximately $60 \mu \mathrm{m}$. This delay ensures the particle will have completely left the $635 \mathrm{~nm}$ beam before excitation with UV, so as to avoid contamination of the detected fluorescence signals with elastically scattered $635 \mathrm{~nm}$ radiation.

The illumination from the xenon arc is imaged onto a $2 \mathrm{~mm} \times 0.8 \mathrm{~mm}$ aperture to remove extraneous light and then refocused via a UV bandpass filter ${ }^{11}(250-300 \mathrm{~nm})$ onto an area approximately $2.2 \mathrm{~mm} \times 1.0 \mathrm{~mm}$ encompassing the intersection of the particle flow and cw $635 \mathrm{~nm}$ beam. The UV fluence at this point produced by the xenon flash is approximately $500 \mu \mathrm{J} / \mathrm{cm}^{2}$. The FWHM of the xenon pulse is approximately $2.5 \mu \mathrm{s}$ and particle fluorescence produced by this excitation is similarly reflected by the ellipsoidal reflector onto the dichroic beam-splitter. The FL2 channel thus records fluorescence in the band $\sim 400-650 \mathrm{~nm}$ (the upper limit set by the PMT detector roll-off) coincident with the NADH emission maximum, whilst light reflected by the beamsplitter passes through a 320nm highpass filter (Biral $F L 1^{11}$ ) and onto the FL1 detector. FL1 thus records fluorescence in the $320-400 \mathrm{~nm}$ band, coincident with the majority of the tryptophan emission peak.

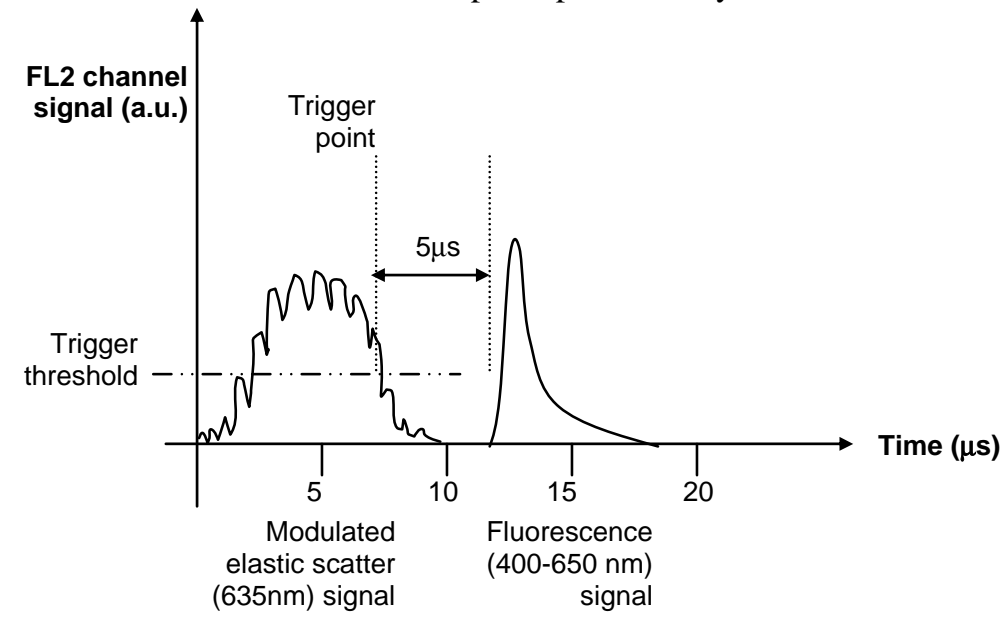

Fig.10. Signal sequence recorded by FL2 detector channel. 
It is, of course, possible that in high aerosol concentrations a second particle could enter the $635 \mathrm{~nm}$ beam when the xenon fires. The signal detected by the FL2 channel would thus comprise fluorescence from both particles plus elastic scatter from the particle in the $635 \mathrm{~nm}$ beam. In order to be able to detect these unwanted events, the cw $635 \mathrm{~nm}$ beam is analog modulated at $10 \mathrm{MHz}$. If the signal recorded by the FL2 channel contains any element of this modulation (detected by the subsequent electronics) the recorded data for that 'coincident-particle' event is ignored.

Whilst this prototype sensor is designed to record single particle fluorescence, it is rate limited by the re-charge rate (and heat dissipation limits) of the xenon source. With the current discharge capacitor employed, this limit is approximately 100 flashes/second, although modification to raise this to 200 /second is possible. To extend xenon lifetime to greater than 6 months, it is envisaged that the sensor would operate in the following manner: All particles passing through the $\mathrm{cw} 635 \mathrm{~nm}$ laser beam would be registered in terms of size (proportional to scatter signal) and number (up to a maximum of $\sim 5,000$ /second). For particles deemed by the trigger threshold to exceed $\sim 0.5 \mu \mathrm{m}$ in size, the xenon would fire and subsequent FL1 and FL2 fluorescence data for those particles recorded. In 'steady-state' mode, this flash rate would be limited to 50 per second. Should the recorded fluorescence data indicate the presence of an abnormally high proportion of potential biological particles, the controlling electronics would temporarily raise the xenon pulse rate to $\sim 200$ /second in order to improve the statistical validity of the assessment. As a guide: if the ambient aerosol concentration of particles greater than the $0.5 \mu \mathrm{m}$ size threshold was $\sim 5 \times 10^{4} /$ litre, and the xenon was operating at a rate of 50 flashes per second, then fluorescence data would be recorded from approximately $50 \%$ of all particles.

Completion of the prototype is scheduled for early Autumn 2004.

\section{REFERENCES}

1. R.G. Pinnick, S.C. Hill, P. Nachman, J.D. Pendleton, G.L. Fernandez, M.W. Mayo, and J.G. Bruno; "Fluorescent particle counter for detecting airborne bacteria and other biological particles”. Aerosol Sci. Technol. 23 (4): $653-$ 6641995.

2. P.P. Hairston, J. Ho, and F.R. Quant; "Design of an instrument for real-time detection of bioaerosols using simultaneous measurement of particle aerodynamic size and intrinsic fluorescence”. J. Aerosol Sci. 28 (3): 471-480, 1997.

3. G. Chen, P. Nachman, R. G. Pinnick, S. C. Hill, and R. K. Chang, "Conditional-firing aerosol-fluorescence spectrum analyzer for individual airborne particles with pulsed 266-nm laser excitation,” Opt. Lett. 21, 1307-1309 1996.

4. M. Seaver, J. D. Eversole, J. J. Hardgrove, W. K. Cary, Jr., and D. C. Roselle, "Size and fluorescence measurements for field detection of biological aerosols,” Aerosol Sci. Technol. 30, 174-185 1999.

5. F.L. Reyes, T. H. Jeys, N. R. Newbury, C. A. Primmerman, G. S. Rowe, and A. Sanchez. "Bio-aerosol Fluorescence Sensor”. Field Anal. Chem. and Technol. 3(4-5): 240-248 1999.

6. See: http://www.darpa.mil/mto/suvos/

7. T.H. Jeys, L. Desmarais, E. J. Lynch, and J.R. Ochoa; “Development of a UV LED based Biosensor”. Sensors and Command, Control, and Intelligence Technologies for Homeland Defense and Law Enforcement. Ed: E.M. Carrapezza. SPIE 5071: 234-240 2003.

8. P.H. Kaye, J.E. Barton , E. Hirst , and J.M. Clark; "Simultaneous Light Scattering and Intrinsic Fluorescence Measurement for the Classification of Airborne Particles”. Applied Optics 39, 21: 3738-3745 2000.

9. See: http://optoelectronics.perkinelmer.com/content/RelatedLinks/rsl3100.pdf

10. See: http://www.djoptecs.co.kr/filter.asp?gubun=05

11. The Xenon 1 excitation filter and FL1 fluorescence filter were provided by Bristol Industrial \& Research Associates Ltd (BIRAL), Portishead, UK. 